\title{
Editorial
}

\section{Very Heavy Quarks at the LHC}

\author{
Johan Alwall, ${ }^{1}$ Kai-Feng Chen, ${ }^{2}$ Tao Han, ${ }^{3}$ and George Wei-Shu Hou ${ }^{2}$ \\ ${ }^{1}$ Theoretical Physics Department, Fermi National Accelerator Laboratory, Batavia, IL 60510, USA \\ ${ }^{2}$ Department of Physics, National Taiwan University, Taipei 10617, Taiwan \\ ${ }^{3}$ Department of Physics \& Astronomy, University of Pittsburgh, Pittsburgh, PA 15260, USA
}

Correspondence should be addressed to George Wei-Shu Hou; wshou@phys.ntu.edu.tw

Received 9 September 2013; Accepted 9 September 2013

Copyright (C) 2013 Johan Alwall et al. This is an open access article distributed under the Creative Commons Attribution License, which permits unrestricted use, distribution, and reproduction in any medium, provided the original work is properly cited.

Ever since the emergence of the third generation of fermions in the 1970s, which was only completed in 1995 with the discovery of the top quark, the heaviest fermion known to date, the question has lingered: can there be more fermion generations in Nature, that is, a 4th generation? By this one means a repetition of the left-handed (weak) doublet and right-handed singlet pattern that is observed for the first three generations.

The interest was dampened, however, by electroweak precision tests as well as neutrino counting, both done at LEP. By late 1990s, when neutrinos were established to have a tiny mass, one knew already that the 4 th generation would be special if it existed at all, in that the neutral lepton (rather than "neutrino") sector would have to be different.

This special volume concerns quarks only and will not be much concerned with the lepton sector, and we know beforehand that the new heavy chiral quarks would have to be very heavy. But "the 4th generation was declared dead many times ... why were there so many prejudices against the existence of this simple model?" Despite these prejudices, there was a visible surge in interest in the 4th generation by 2010-2011, as a confluence of flavor and CP violation studies, as well as direct search, where the mantle has been transferred from the Tevatron (and B factories) to the LHC. We have witnessed tremendous progress in particle physics during 2011-2012 because of the successful 7 and $8 \mathrm{TeV}$ run program of the LHC. Intriguingly, this special volume both enjoyed but also suffered as a victim of this success.

The paper by A. Lenz best captures the suffering of the 4 th generation from the success of the LHC. In fact, our previous quotes were from this review paper, which moved on to state that LHC data "exclude the possibility of having simply a perturbative fourth generation of fermions with one Higgs doublet (SM4)." This is mostly due to the emergence of the $126 \mathrm{GeV}$ boson that fits the Higgs boson of SM well, while the expected order of magnitude enhanced cross-section with SM4 is not observed. Despite this, and partially alluded to by the Lenz paper, the paper by S. Bar-Shalom et al. took account of the $126 \mathrm{GeV}$ boson and explored it beyond SM4, by considering two Higgs doublets. A somewhat different view was taken up in the paper by P. Q. Hung, exploring strong Yukawa coupling induced fermion-antifermion condensation, the possibility of scale-invariance and "quasi-fixed point" above $\mathrm{TeV}$ scale, and the emergence of multi-Higgs doublets. A third view is reported in the paper by W.-S. Hou, which explores strong Yukawa-induced quark-antiquark condensation through a "bootstrap gap equation" involving the Goldstone boson self-consistently but at the cost of assuming the $126 \mathrm{GeV}$ boson to be the dilaton of scale invariance violation. Probably none of these approaches carry the full truth, but whether the 4th generation can survive beyond SM4 in some form would have to await more data. In this vein, it is also worth mentioning the paper by J. Bulava et al., which makes a comprehensive (but yet unfinished) study of the phase structure of the Higgs-Yukawa model on the lattice. This is the genuine nonperturbative approach and is of at least mathematical interest regardless of phenomenological outcome at the LHC.

Reflecting the interest in the 4th generation prior to the discovery of the $126 \mathrm{GeV}$ boson, the paper by E. Tsedenbaljir explores the possible formation of bound states through strong Yukawa coupling; the paper by J. Alwall and J. Tandean explores the possible, intriguing connection with dark matter; 
and the paper by W.-S. Hou and M. Kohda makes the preliminary study of the link with electroweak baryogenesis, as the much enhanced strength of $\mathrm{CP}$ violation (fit for baryogenesis) remains a rationale for still keeping in mind a 4th generation of quarks.

But we certainly have to admit that the discovery of the $126 \mathrm{GeV}$ (better than) Higgs-like boson excludes the 4th generation once again if not once and for all. The possibility of nonchiral representations, however, is not excluded; the paper by Y. Okada and L. Panizzi explores the phenomenology of vector-like quarks, which is now the mainstream for new heavy quarks. There are also other efforts that do not depend on the chiral nature of heavy quark search. One is the search for long-lived heavy quarks discussed in the paper by M. Buchkremer and A. Schmidt, and another is the brief review by A. Giammanco of single top electroweak production as a probe of possible new heavy quarks. All these topics continue to be of great interest.

With the biggest regret, we were not successful in getting an experimental review on the status of new heavy quark search with 2011-2012 LHC data. The experimental search was too volatile on the frontline, such that the committed authors were too occupied to complete the review in time, which again reflects the success and great progress of the LHC program. In the same vein, we did not succeed in getting a review paper on deeper theories involving vector-like quarks, the one that survived the emergence of the light Higgs boson. However, we think the articles of this volume as described above succeed in giving a snapshot on the first phase of very heavy quark studies at the LHC.

Johan Alwall

Kai-Feng Chen

Tao Han

George Wei-Shu Hou 

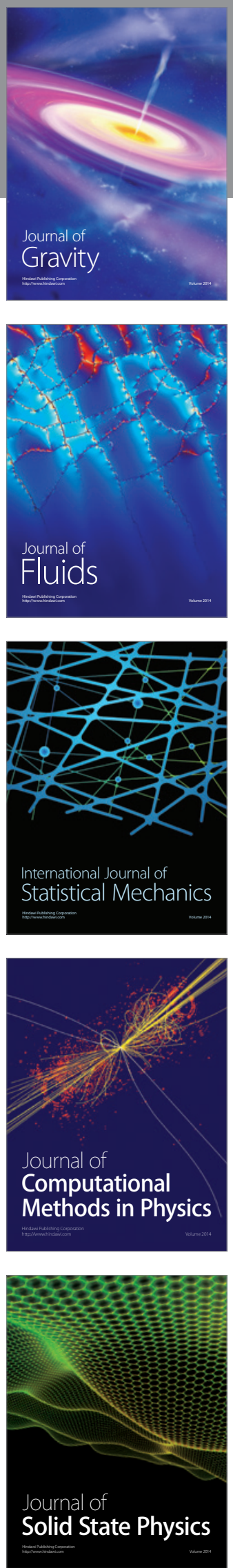

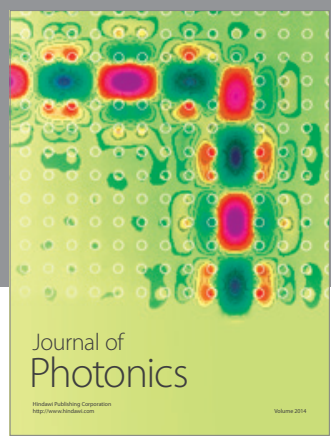

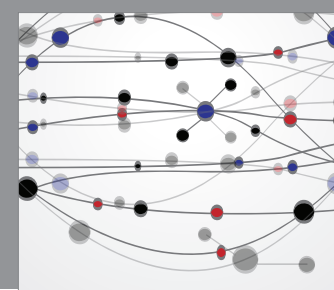

The Scientific World Journal

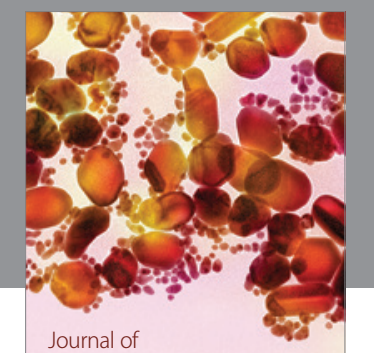

Soft Matter
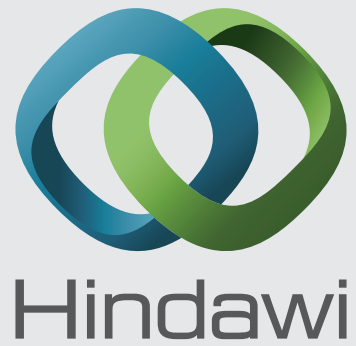

Submit your manuscripts at

http://www.hindawi.com
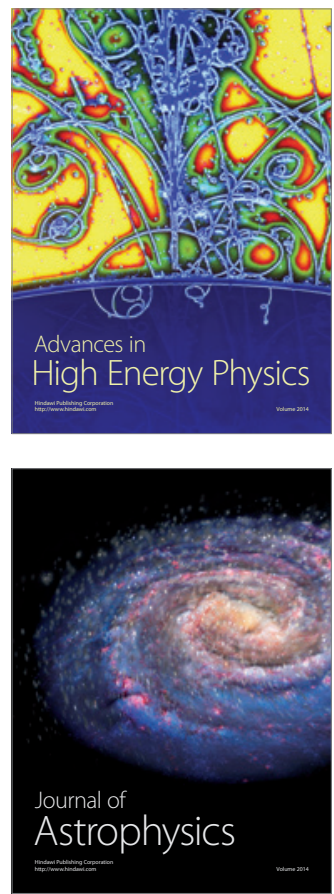
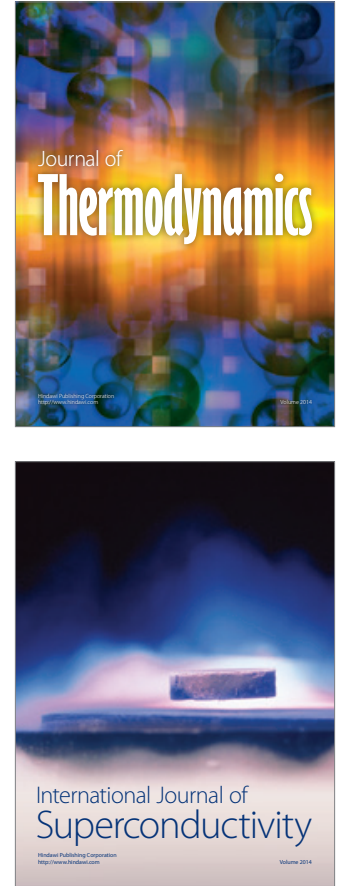
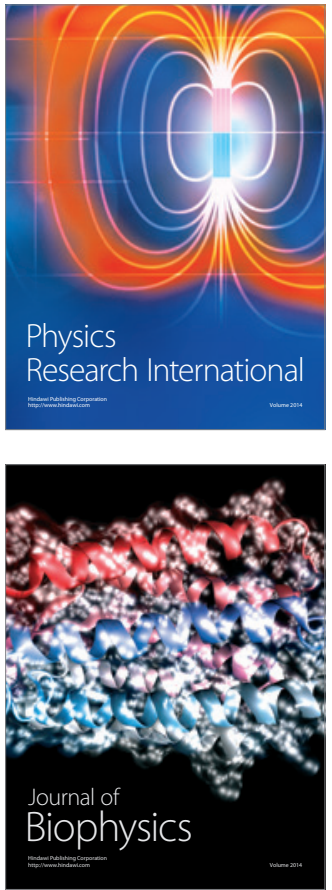
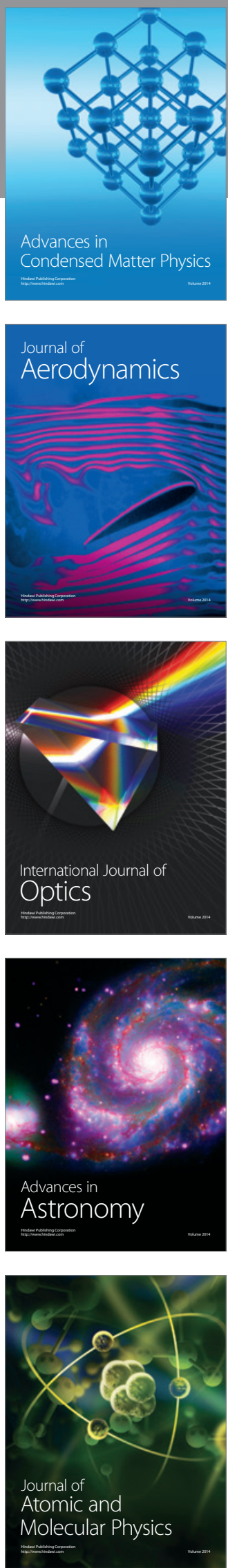